\title{
Coexistence of magnetic and electrical order in the new perovskite-like $\left(\mathrm{C}_{3} \mathrm{~N}_{2} \mathrm{H}_{5}\right)\left[\mathrm{Mn}(\mathrm{HCOO})_{3}\right]$ formate $\uparrow$
}

\section{B. Pato-Doldán ${ }^{a}$, L. C. Gómez-Aguirre ${ }^{a}$,J. M. Bermúdez-García ${ }^{a}$, M. Sánchez-} Andújar $^{a}$, A. $\quad$ Fondado $^{b}$, J. $\quad$ Mira $^{b}$, S. Castro-García ${ }^{a}$ and M. A. SeñarísRodríguez $^{* a}$

${ }^{a}$ Department of Fundamental Chemistry, University of A Coruña, 15071 A Coruña, Spain. Email: m.senaris.rodriguez@udc.es; $\quad$ Fax: $\quad+34$ 981167065; Tel: $\quad+34981167000$ ${ }^{b}$ Department of Applied Physics, University of Santiago de Compostela, 15782, Santiago de Compostela, Spain

DOI: 10.1039/C3RA43165G (Paper)

RSC Advances, 2013, 3, 22404-22411

Received 24th June 2013, Accepted 16th September 2013

First published on the web 16th September 2013

\begin{abstract}
In this work we further the structural characterization of the recently discovered $\left(\mathrm{C}_{3} \mathrm{~N}_{2} \mathrm{H}_{5}\right)\left[\mathrm{Mn}(\mathrm{HCOO})_{3}\right]$ metal-organic framework with perovskite-like structure, and we present its magnetic and dielectric properties up to $350 \mathrm{~K}$. At low temperature, the $\mathrm{C}_{3} \mathrm{~N}_{2} \mathrm{H}_{5}{ }^{+}$imidazolium cations, that sit oblique within the cavities of the $\left[\mathrm{Mn}(\mathrm{HCOO})_{3}\right]^{-}$framework structure, show a cooperative order resulting in an antiparallel arrangement of their electrical dipole moments. Very interestingly, it is only above $220 \mathrm{~K}$ that thermal energy seems to be able to break this antiferroelectric order, resulting in a linear increase of its dielectric constant with temperature. In addition, this $\mathrm{Mn}$ (II) compound is antiferromagnetic below $T_{\mathrm{N}}=9 \mathrm{~K}$, with a slightly non-collinear arrangement of its magnetic moments, yielding to a weak ferromagnetism. Therefore, this is a new multiferroic material which exhibits coexistence of magnetic and electric ordering.
\end{abstract}




\section{Introduction}

Recently, progress in metal-organic framework (MOF) research has opened up new possibilities to achieve hybrid materials with a rich variety of functional and even multifunctional properties. ${ }^{1}$ Among them, formate-based MOFs with magnetic, ${ }^{2}$ dielectric $^{3}$ and multiferroic properties ${ }^{4,5}$ have been recently reported and are particularly interesting.

From the structural point of view, all metal formate frameworks of formula $\left[\right.$ Amine $\left.\mathrm{H}_{n}\right]\left[\mathrm{M}(\mathrm{HCOO})_{3}\right]_{n}$ (where $\mathrm{M}$ are divalent cations and $\mathrm{AmineH}_{n}{ }^{+}$are different protonated amines) consist of six-connected nodes of octahedral $\left[\mathrm{MO}_{6}\right]$ units, linked by bridging $\mathrm{HCOO}^{-}$anions, with the $\mathrm{AmineH}_{n}{ }^{+}$cations located in the resulting cavities. ${ }^{2}$ Depending on the size of the amine cations, different framework topologies have been reported. For example, in the presence of the smallest cations (such as ammonium) the metal cation connects to its six neighboring metal ions in an arrangement of a trigonal prism leading a $\left(4^{9} \cdot 6^{6}\right)$ topology. ${ }^{6}$ Meanwhile mid-sized alkylammonium cations, such as methylammonium, ethylammonium, dimethylammonium and cyclotrimethyleneammonium (or azetidinium cation), lead to a perovskite-like structure, where the metal cation has its six neighbours arranged in an octahedron, resulting in a $\left(4^{12} \cdot 6^{3}\right)$ topology. ${ }^{7,8}$ Larger linear protonated di-, tri-, and tetra-amines (such as $\mathrm{N}, \mathrm{N}$-dimethylethylenediamine) lead to the formation of novel bi-nodal metal formate frameworks with $\left(4^{12} \cdot 6^{3}\right)\left(4^{9} \cdot 6^{6}\right)_{n-1}$ topologies. ${ }^{9}$

Some of these compounds display very interesting functional properties. For example, it has been reported that several dimethylammonium metal formates with perovskite-like structure and formula $\left[\left(\mathrm{CH}_{3}\right)_{2} \mathrm{NH}_{2}\right]\left[\mathrm{M}(\mathrm{HCOO})_{3}\right]\left(\mathrm{M}=\mathrm{Mn}^{2+}, \mathrm{Fe}^{2+}, \mathrm{Co}^{2+}, \mathrm{Ni}^{2+}, \mathrm{Zn}^{2+}\right.$ and $\left.\mathrm{Mg}^{2+}\right)$, are switchable dielectrics which undergo transitions between high and low dielectric states, related to the appearance of electrical order, in the temperature range of $160-185 \mathrm{~K}$ in the case of the first five $\mathrm{M}^{2+}, 3,4,10$ or even at $270 \mathrm{~K}$ (almost room temperature) in the case of $\mathrm{Mg}^{2+}$ compound. ${ }^{11}$ Even more, such compounds (except the $\mathrm{Zn}^{2+}$ - and $\mathrm{Mg}^{2+}$-ones) are examples of MOFs with multiferroic behaviour, ${ }^{4}$ as they also exhibit weak ferromagnetism at low temperatures $\left(T_{\mathrm{C}}=8-\right.$ $36 \mathrm{~K}) .^{12}$

Other formates with perovskite architecture and containing different alkylammonium cations, such as the azetidinium cation in $\left[\left(\mathrm{CH}_{2}\right)_{3} \mathrm{NH}_{2}\right]\left[\mathrm{Cu}(\mathrm{HCOO})_{3}\right],{ }^{13}$ or the guanidinium cation in $\left[\mathrm{C}\left(\mathrm{NH}_{2}\right)_{3}\right]\left[\mathrm{Cu}(\mathrm{HCOO})_{3}\right]$ (ref. 14) are also attracting attention in view of giant dielectric anomalies ${ }^{13}$ and magnetoelectric multiferroicity, ${ }^{14}$ respectively.

Very recently, a new perovskite like manganese formate, containing the five-membered ring imidazolium cation, has been reported. ${ }^{15}$ From the structural point of view, it has been described that this $\left(\mathrm{C}_{3} \mathrm{~N}_{2} \mathrm{H}_{5}\right)\left[\mathrm{Mn}(\mathrm{HCOO})_{3}\right]$ compound displays an order-disorder transition at around 435 
$\mathrm{K}$ from an ordered monoclinic phase to a tetragonal one. In this latter, both the framework and the guest imidazolium cation are disordered. Meanwhile, the room temperature structure is ordered and consists of two crystallographically independent $\mathrm{HIm}^{+}$cations and two crystallographically independent $\mathrm{Mn}^{2+}$ ions.

The aim of this work is to deepen in the structural characteristics of the low-temperature phase of this new perovskite-type compound $\left(\mathrm{C}_{3} \mathrm{~N}_{2} \mathrm{H}_{5}\right)\left[\mathrm{Mn}(\mathrm{HCOO})_{3}\right]$, that can be different from those shown at room temperature, and to study its magnetic and dielectric properties.

Our choice and interest in this material relies on two factors: (i) the fact that it is a very promising candidate to present interesting dielectric properties, (ii) the possibility of finding cooperative magnetism, as the preservation of the $3 \mathrm{D}\left[\mathrm{Mn}(\mathrm{HCOO})_{3}\right]$ perovskite framework favors long range magnetic interactions among the divalent manganese cations. With these two ingredients this compound is a very good candidate to be a new multiferroic material with coexistence of magnetic and electrical order.

\section{Experimental section}

\section{Materials}

$\mathrm{MnCl}_{2} \cdot 4 \mathrm{H}_{2} \mathrm{O}$ (99.9\%, Fluka), imidazole (99.5\%, Aldrich), absolute methanol (Panreac) and formic acid (98\%, Fluka) were commercially available and used as purchased without further purification.

\section{Synthesis}

The synthetic route used here is an adaptation of the previously reported method for the preparation of the $[\mathrm{G}]\left[\mathrm{Mn}(\mathrm{HCOO})_{3}\right]$ compounds, ${ }^{7}$ where the synthesis of the metal formate frameworks is templated by alkylammonium cations, and accomplished through a mild solution chemistry method at ambient temperature, by employing suitable metal salts and amines neutralized by formic acid. It differs from the one just described for the preparation of the $\left(\mathrm{C}_{3} \mathrm{~N}_{2} \mathrm{H}_{5}\right)\left[\mathrm{Mn}(\mathrm{HCOO})_{3}\right]$ compound $^{15}$ in the starting materials and solvent used, and it allows to obtain the desired compound as a single phase material.

In a typical experiment $2.5 \mathrm{ml}$ of a methanol solution of $0.5 \mathrm{M}$ formic acid $\mathrm{HCOOH}$ and 0.5 $\mathrm{M}$ imidazole $\mathrm{C}_{3} \mathrm{~N}_{2} \mathrm{H}_{4}$ were placed at the bottom of a glass tube. Upon the $\mathrm{HCOOH}-$ $\mathrm{C}_{3} \mathrm{~N}_{2} \mathrm{H}_{4}$ solution, $1 \mathrm{ml}$ of methanol was carefully added, followed by carefully layering $4 \mathrm{ml}$ of a methanol solution of $0.10 \mathrm{M} \mathrm{MnCl}_{2} \cdot 4 \mathrm{H}_{2} \mathrm{O}$. The tube was sealed and kept undisturbed. Tiny uncolored square-shaped crystals appeared after two days. They were collected, washed with ethanol and dried at room temperature. They were found to be stable in air. 


\section{Crystallographic structure determination}

Single-crystal data sets were collected at $295 \mathrm{~K}$ and $100 \mathrm{~K}$ in a Bruker-Nonius x8 ApexII X-ray diffractometer equipped with a CCD detector and using monochromatic $\operatorname{MoK} \alpha_{1}$ radiation $(\lambda=$ $0.71073 \AA$ A). A suitable crystal of the sample was chosen and mounted on a glass fiber using instant glue. For the $100 \mathrm{~K}$ set, the crystal temperature was maintained using a cold stream of nitrogen from a Kyroflex cryostream cooler. The data integration and reduction was performed using the Apex2 V.1.0-27 (BrukerNonius, 2005) suite software. The intensity collected was corrected for Lorentz and polarization effects and for absorption by semiempirical methods on the basis of symmetry-equivalent data using SADABS (2004) of the suite software. The structures were solved by the direct method using the SHELXS-97 (ref. 16) program and were refined by least squares method on SHELXL-97 (ref. 17).

To solve the structure at $295 \mathrm{~K}$ and $100 \mathrm{~K}$, anisotropic thermal factors were employed for the non- $\mathrm{H}$ atoms. The hydrogen atoms of the formate and imidazolium ions were found in the Fourier map and their coordinates and isotropic thermal factors were refined.

In addition, the compound was studied by X-ray powder diffraction (XRPD) at room temperature in a Siemens D-5000 diffractometer using CuKa radiation $(\lambda=1.5418 \AA)$. The XRPD pattern was analyzed by the Le Bail profile analysis using the GSAS software. ${ }^{18}$

\section{Thermal studies}

Differential Scanning Calorimetric (DSC) analyses were carried out in a TA Instruments MDSC Q-2000 with a liquid nitrogen cooling system, by cooling and heating the sample at different rates $\left(2-10^{\circ} \mathrm{min}^{-1}\right)$ from $300 \mathrm{~K}$ up to $100 \mathrm{~K}$ under nitrogen atmosphere.

\section{Raman spectroscopy}

Powder Raman spectra were recorded at different temperatures within the temperature range [350-100] K (namely at $350 \mathrm{~K}, 300 \mathrm{~K}, 220 \mathrm{~K}, 180 \mathrm{~K}, 120 \mathrm{~K}$ and $100 \mathrm{~K}$ ) using a Renishaw inVia Raman microscope equipped with a CCD camera. Spectra were recorded over the wavenumber range of $50-3200 \mathrm{~cm}^{-1}$ using the linearly polarized $784 \mathrm{~nm}$ line of diode laser (with power of $150 \mathrm{~mW}$ ), focused to a $65 \mu \mathrm{m}$ spot through a $50 \times$ microscope objective onto the sample surface. A microcryostat from Linkam scientific instruments was used for the low temperature studies. 


\section{Dielectric properties}

The complex dielectric permittivity $\left(\varepsilon_{\mathrm{r}}=\varepsilon_{\mathrm{r}}^{\prime}-i \varepsilon_{\mathrm{r}}^{\prime \prime}\right)$ of the cold-press pelletized sample was measured as a function of frequency and temperature with a parallel-plate capacitor coupled to a Solartron1260A Impedance/Gain-Phase Analyzer, capable to measure in the frequency range from $10 \mu \mathrm{Hz}$ up to $32 \mathrm{MHz}$ using an amplitude of $2 \mathrm{~V}$. The capacitor was mounted in a Janis SVT200T cryostat refrigerated with liquid nitrogen, and with a Lakeshore 332 incorporated to control the temperature from $78 \mathrm{~K}$ up to $400 \mathrm{~K}$. The data were collected on heating.

Pelletized samples made out of cold-press non-oriented single crystal with an area of approximately $530 \mathrm{~mm}^{2}$ and a thickness of approximately $1.3 \mathrm{~mm}$ were prepared to fit into the capacitor, and gold was sputtered on their surfaces to ensure a good electrical contact with the electrodes.

All the dielectric measurements were carried out in a nitrogen atmosphere where several cycles of vacuum and nitrogen gas were performed to ensure that the sample environment is free of water.

The impedance analysis software SMART (Solartron Analytical) was used for data acquisition and processing. Impedance complex plane plots were analyzed using the LEVM program, a particular program for complex nonlinear least squares fitting. ${ }^{19}$

Polarization $(P) v s$. electric field $(E)$ measurements were carried out using a home-built setup based on a Sawyer-Tower circuit where the hysteresis loops were recorded using a Keithley 6517B electrometer.

\section{Magnetic properties}

The magnetic properties were studied in a Quantum Design MPMS SQUID magnetometer on a collection of small single crystals randomly oriented in the magnetic field. Zero-field-cooled (ZFC) and field-cooled (FC) magnetic susceptibility data were obtained under different magnetic fields (50 Oe, $100 \mathrm{Oe}$ and $1000 \mathrm{Oe}$ ) in the temperature range [5-300] K. Hysteresis loops in ZFC conditions were obtained at $5 \mathrm{~K}$ varying the magnetic field up to $\pm 50 \mathrm{kOe}$. The experimental susceptibilities were corrected for the diamagnetism of the constituent atoms (using the Pascal's tables) ${ }^{20}$ and an experimental correction for the sample holder was applied. 


\section{Results and discussion}

\section{Crystallographic structure}

Single-crystal X-ray studies carried out at $295^{\S}$ and at $100 \mathrm{~K}^{\ddagger}$ reveal that, at both temperatures, $\left(\mathrm{C}_{3} \mathrm{~N}_{2} \mathrm{H}_{5}\right)\left[\mathrm{Mn}(\mathrm{HCOO})_{3}\right]$ shows a distorted perovskite-like structure (see Fig. 1) with monoclinic symmetry (space-group $\left.P 2_{1} / n, Z=8\right)$, and very similar cell parameters $\left(a_{295 \mathrm{~K}}=12.3034(4)\right.$ $\AA, b_{295 \mathrm{~K}}=12.4408(4) \AA, c_{295 \mathrm{~K}}=12.8156(4) \AA$ and $\beta_{295 \mathrm{~K}}=91.190(2)^{\circ} v s . a_{100 \mathrm{~K}}=12.1962(3)$ $\AA, b_{100 \mathrm{~K}}=12.3675(3) \AA, c_{100 \mathrm{~K}}=12.8155(4) \AA$ and $\left.\beta_{100 \mathrm{~K}}=91.428(1)^{\circ}\right)$. The former are in good agreement with those recently reported for this compound. ${ }^{15}$
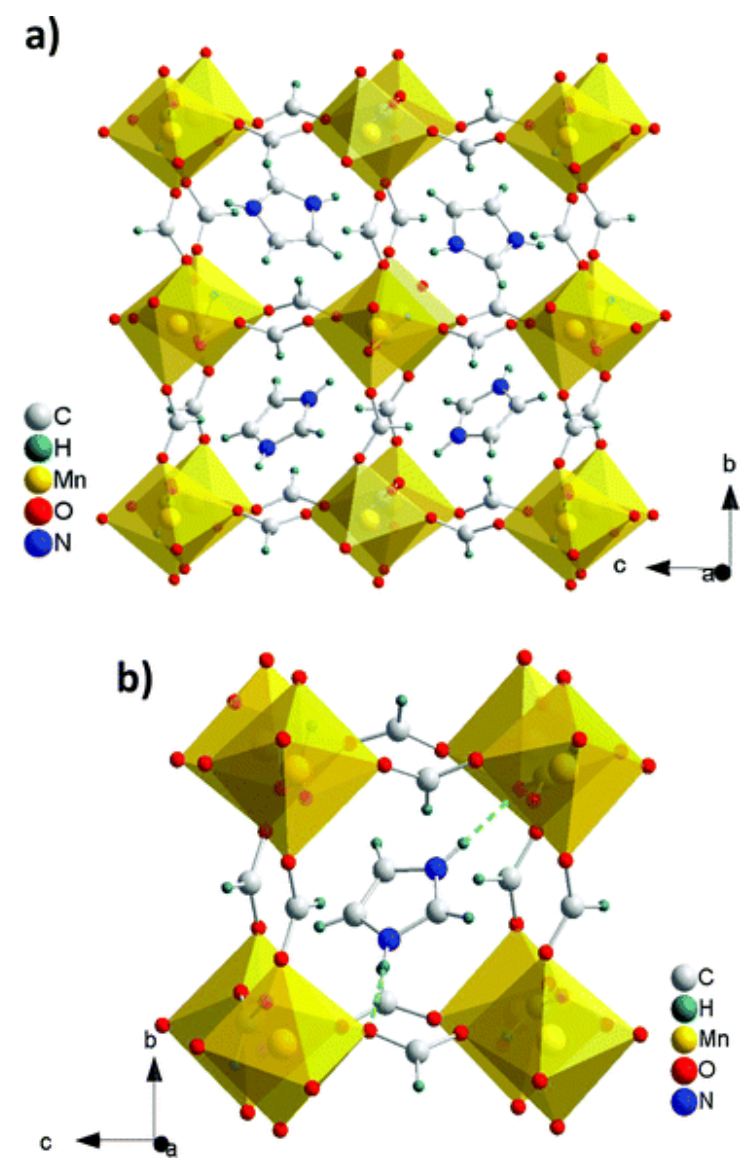

Fig. 1 Crystal structure of the $\left(\mathrm{C}_{3} \mathrm{~N}_{2} \mathrm{H}_{5}\right)\left[\mathrm{Mn}(\mathrm{HCOO})_{3}\right]$ compound: (a) general view showing the cooperative ordering of the imidazolium cations; (b) detail showing the H-bonds, as dashed green lines, between the $\mathrm{N}-\mathrm{H}^{\cdots} \mathrm{O}$ atoms.

As already indicated there,${ }^{15}$ the asymmetric unit of the structure contains two independent manganese cations, six $\mathrm{HCOO}^{-}$formate groups bonded to the metal cations, and two $\mathrm{C}_{3} \mathrm{~N}_{2} \mathrm{H}_{5}{ }^{+}$imidazolium cations. As a result, the two crystallographically different $\mathrm{Mn}$ cations are connected to its six nearest neighbors metal ions through six formate bridges in a distorted octahedral environment with six different $\mathrm{Mn}-\mathrm{O}$ distances, that change very little with temperature, as it is also the case for the whole $\left[\mathrm{Mn}\left(\mathrm{HCOO}_{3}\right)\right]^{-}$framework (see Table SI of ESI $\dagger$ ). 
We have quantified the magnitude of these $\left[\mathrm{MnO}_{6}\right]$ octahedral distortions by taking into account the six $\mathrm{Mn}-\mathrm{O}$ bond lengths and the three trans $\mathrm{O}-\mathrm{Mn}-\mathrm{O}$ bond angles. ${ }^{21}$ Thus we were able to calculate the out-of-center distortion $\left(\Delta_{\mathrm{d}}\right)$ values of 0.09 and 0.1 , for the $\left[\mathrm{Mn}(1) \mathrm{O}_{6}\right]$ and $\left[\mathrm{Mn}(2) \mathrm{O}_{6}\right]$ octahedra respectively, indicating a weak octahedral distortion. ${ }^{20}$ As for their direction, the $\operatorname{Mn}(1)$ cation is slightly shifted towards one of the edges of the octahedra while the $\operatorname{Mn}(2)$ cation is displaced towards one of the faces of the octahedra. It is worth noting that, in the resulting centrosymmetric crystal structure, their associated small dipole moments compensate each other and do not give rise at a net polarization.

It should also be indicated that these manganese cations show the expected formal valence of $\sim 2$, as estimated by the bond-valence sum (BVS) method (see Table SI of ESI $\dagger$ ).

In this low-temperature phase, the eight imidazolium cations of the monoclinic unit cell (that occupy two distinguishable crystallographically positions, and form H-bonds to the framework, as already described in ref. 15, and represented in Fig. 1b), are cooperatively ordered inside the pseudo-cubo octahedral cavities (see Fig. 1a); and their molecular plane lies nearly parallel to, alternatively, the (111) and the (-111) planes of the conventional perovskite-like cell (see Fig. 1b), resulting in an antiparallel arrangement of their associated electrical dipole moments.

In addition, and very interestingly, the data obtained at room temperature and at $100 \mathrm{~K}$ reveal small but meaningful differences concerning the imidazolium cations and their interaction with the framework as a function of temperature. In this context and as it can be seen in Table SI and Fig. S1 of ESI, $\uparrow$ there are significant changes in the intra-imidazolium cationbond lengths and particularly in the distance between the $\mathrm{N}$ atoms of the imidazolium cation and the $\mathrm{O}$ atoms of the closest formate anions, that are shorter at low temperature. In any case, at both temperatures these distances $\left(d_{(\mathrm{N}-\mathrm{O})} \sim 2.75-2.84 \AA\right.$ ) are short enough to allow the presence of two bridging $\mathrm{H}$-bond between such two $\mathrm{N}-\mathrm{O}$ atoms, even if they will be stronger at low temperatures. Additionally, at room temperature the thermal displacements of all the $\mathrm{C}, \mathrm{N}$ and $\mathrm{H}$ atoms of the imidazolium cations are significantly larger than those at low temperature (see Fig. S1 of ESI $\dagger$ ). This behaviour may be explained in terms of the presence of already a certain degree of dynamical motion of the entire imidazolium cation at room temperature, (as also found in the Raman studies and dielectric measurements, see below), process that can be thermally activated, and that is relatively common in other compounds containing weakly bound imidazolium cations. ${ }^{22-25}$ Nevertheless these cations would remain essentially ordered and linked to the framework and their complete disorder will only be achieved above room temperature, namely at $T>435 \mathrm{~K} .^{15}$ 
It is worth noting that the Le Bail refinement of the XRPD data obtained at room temperature confirms that the Mn-compound is single-phased, showing good agreement between the experimental data and the proposed model for the fitting (see Fig. S2 of ESI + ).

\section{Thermal properties}

We have carried out low temperature DSC measurements on this compound paying specially attention to temperatures around $200 \mathrm{~K}$, where an anomaly was detected in the dielectric measurements (see below). Nevertheless, and as it can be seen in Fig. S3 of ESI $\uparrow$ the DSC curve does not show any peak or anomaly in the whole temperature studied range.

\section{Raman studies}

To obtain more information about possible dynamic processes involving the imidazolium cations in the low-temperature phase, we have recorded Raman spectra at different temperatures (Fig. 2 and Fig. S4 of ESI†े), as vibrational spectroscopies are known to be very useful tools to study such cases. ${ }^{21-23}$

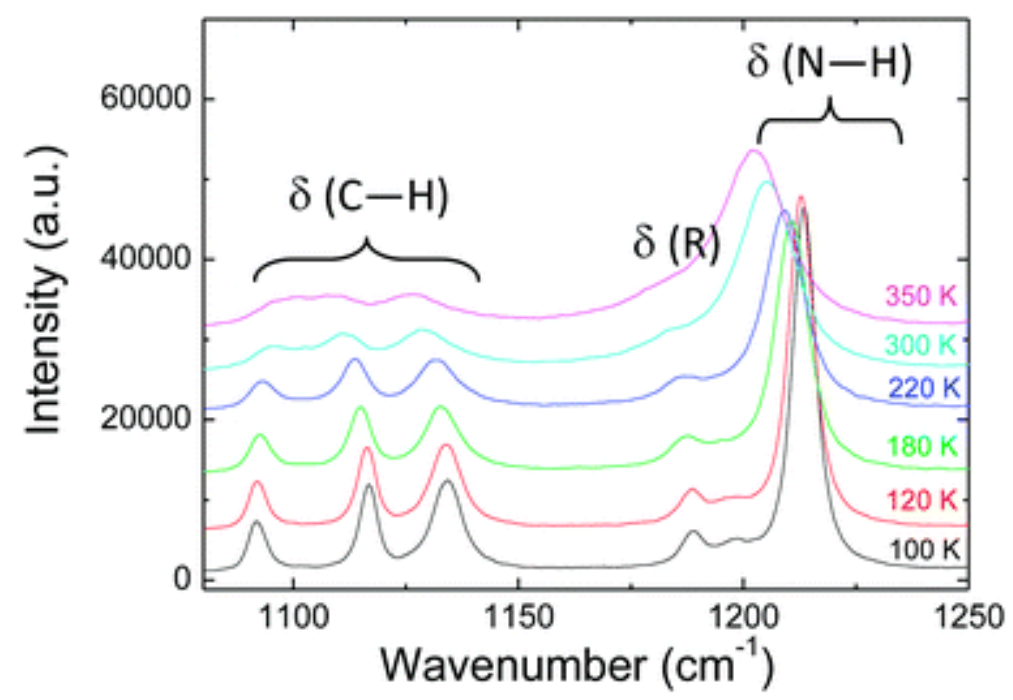

Fig. 2 Detail of the Raman spectra of the $\left(\mathrm{C}_{3} \mathrm{~N}_{2} \mathrm{H}_{5}\right)\left[\mathrm{Mn}(\mathrm{HCOO})_{3}\right]$ compound in the lowwavenumber region and recorded at different temperatures in the interval [100-350] K.

The suggested complete assignment of the observed frequencies, made on the basis of the literature data concerning a formate perovskite ${ }^{10}$ and different imidazolium compounds, ${ }^{21-23} \mathrm{can}$ be seen in Table SII of ESI. $\dagger$

Very interestingly, changes as a function of temperature are observed in the frequency, splitting and intensities of certain peaks ascribed to vibrations of the imidazolium cations, while no significant changes are observed in those peaks attributed to the formate anions. And as it also occurs in the case of the imidazolium halogenoantimonates (III) and bismuthates (III), ${ }^{21-}$ ${ }^{23}$ the most important changes in the Raman spectra of this $\left(\mathrm{C}_{3} \mathrm{~N}_{2} \mathrm{H}_{5}\right)\left[\mathrm{Mn}(\mathrm{HCOO})_{3}\right]$ compound 
are seen to occur in the wavenumber region $1000-1250 \mathrm{~cm}^{-1}$ (see Fig. 2) in the region of deformation vibrations of the imidazolium cations: deformations of the nitrogen-hydrogen bonds $\delta(\mathrm{N}-\mathrm{H})$, of the carbon-hydrogen bonds $\delta(\mathrm{C}-\mathrm{H})$ and also of the whole ring $\delta(\mathrm{R})$. As shown in Fig. 2, close to room temperature the deformation of the modes $\delta(\mathrm{N}-\mathrm{H}), \delta($ ring) and $\delta(\mathrm{C}-\mathrm{H})$ are observed as broad bands while at low temperature they appear as sharp lines.

This finding suggests again a certain change in the degree of freedom of the imidazolium cations inside the perovskite cavity as a function of temperature and reinforce the single crystal $\mathrm{X}$-ray diffraction results. Therefore the imidazolium cations would be completely frozen out and constraint for $T<220 \mathrm{~K}$; meanwhile for $220 \mathrm{~K}<T<300 \mathrm{~K}$ they would have a certain degree of freedom inside the cavities even if they would remain essentially ordered and linked to the framework at difference with the situation found in other compounds where a complete disorder of this cation is already achieved at room temperature and below. ${ }^{21-24}$

\section{Dielectric properties}

Fig. 3 shows the temperature dependence of the real part of the complex dielectric permittivity (the so-called dielectric constant, $\varepsilon_{\mathrm{r}}^{\prime}$ ) and of the dielectric losses $(\tan \delta)$ of $\left(\mathrm{C}_{3} \mathrm{~N}_{2} \mathrm{H}_{5}\right)\left[\mathrm{Mn}(\mathrm{HCOO})_{3}\right]$ in the temperature interval [100-350] $\mathrm{K}$. As it can be seen, at temperatures below $200 \mathrm{~K}$ the dielectric constant shows a low value, $\varepsilon_{\mathrm{r}}^{\prime} \approx 5$, and it is temperature independent. Nevertheless, around $200 \mathrm{~K}$, the $\varepsilon_{\mathrm{r}}^{\prime} v s$. $T$ curve displays a kink, while the loss tangent goes through a maximum. The position of this kink is shifted slightly to higher temperatures as frequency increases. Then, for $T>200 \mathrm{~K}$ the value of $\varepsilon_{\mathrm{r}}^{\prime}$ increases almost linearly with temperature.

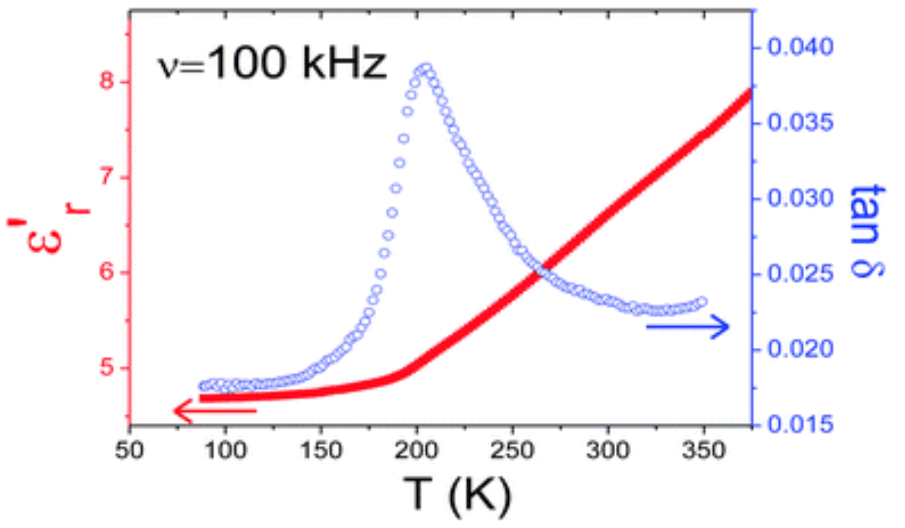

Fig. 3 Temperature dependence of the dielectric constant, $\varepsilon_{\mathrm{r}}^{\prime}$, (solid squares) and the dielectric losses, $\tan \delta, \quad$ (open circles) of $\left(\mathrm{C}_{3} \mathrm{~N}_{2} \mathrm{H}_{5}\right)\left[\mathrm{Mn}(\mathrm{HCOO})_{3}\right]$ measured at $v=100 \mathrm{kHz}$.

Taking into account that the presence of extrinsic factors such electronic and/or ionic conductivity could result in the appearance of artifacts in the dielectric measurements, we have made additional studies to make sure that the observed signal really comes from the sample. 
For this purpose, and to avoid misinterpreting results ${ }^{26,27}$ we have performed impedance complex plane $\left(Z^{\prime \prime} v s . Z^{\prime}\right)$ analysis of the data obtained at different temperatures. A typical impedance complex plane plot for this sample in the whole temperature interval [100-350] $\mathrm{K}$ is shown in Fig. 4. This plot shows a single large arc that can be modeled by an equivalent circuit containing two elements connected in parallel: a resistance $(R)$, and a capacitance $(C)$. As this arc intercepts zero and the order of magnitude of its capacitance is of $\mathrm{pF} \mathrm{cm}^{-1}$, it is associated with the material's bulk response. ${ }^{25}$ Taking into account that this is the only contribution present, we conclude that the observed dielectric response is purely intrinsic in the whole temperature interval studied. This result confirms that the observed dielectric transition is real and associated to the material's bulk response.

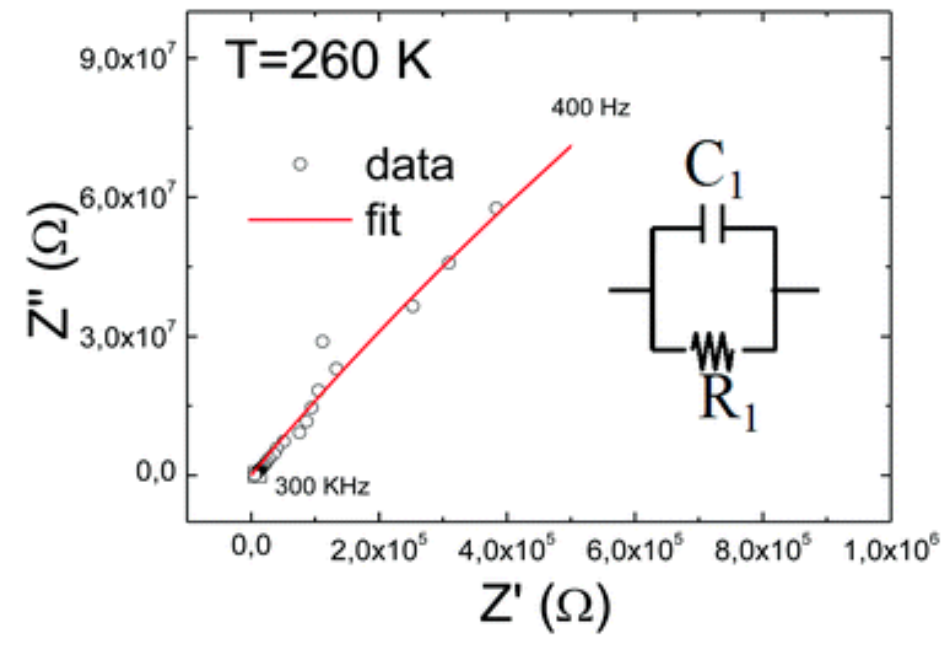

Fig. 4 Example of the typical impedance complex plane plot of $\quad\left(\mathrm{C}_{3} \mathrm{~N}_{2} \mathrm{H}_{5}\right)\left[\mathrm{Mn}(\mathrm{HCOO})_{3}\right]$ observed in the whole temperature and frequency range studied. The open circles represent the experimental data and the solid line represents the fit to the proposed equivalent circuit.

The intrinsic dielectric response of this compound from $350 \mathrm{~K}$ downwards is thus different from that previously reported for other perovskite-like formates with dimethylammonium cations (DMA) inside the cavity ${ }^{3,10}$ and for other compounds such as $[\mathrm{HIm}]_{2}\left[\mathrm{KFe}(\mathrm{CN})_{6}\right]$ with imidazolium cations inside the perovskite cage, ${ }^{24}$ where the dielectric constant shows a sharp jump at a certain transition temperature below room temperature. In this context it is worth noting that such dielectric transitions have been related to order-disorder process of the guest cations. ${ }^{10}$ For example, we have reported that the $\left[\left(\mathrm{CH}_{3}\right)_{2} \mathrm{NH}_{2}\right]\left[\mathrm{Mn}(\mathrm{HCOO})_{3}\right]$ compound displays a paraelectric behaviour for $T>190 \mathrm{~K}$ due to the rotation of the DMA cations inside the cavities and that the dielectric transition observed at $T \approx 190 \mathrm{~K}$ is caused by the freezing of such a molecular rotation upon cooling, with the formation of permanent $\mathrm{H}$-bonds, resulting in a cooperative ordering of the DMA cations. These mechanisms of order-disorder phase transition have been subsequently invoked to explain the dielectric response observed in other $\mathrm{MOFs}^{6 \mathrm{~b}}$ and cage compounds such as $[\mathrm{HIm}]_{2}\left[\mathrm{KFe}(\mathrm{CN})_{6}\right]^{24}$ where the rotation of such cations also experiences a freezing upon cooling around $158 \mathrm{~K}$. 
In the case of the here shown $\left(\mathrm{C}_{3} \mathrm{~N}_{2} \mathrm{H}_{5}\right)\left[\mathrm{Mn}(\mathrm{HCOO})_{3}\right]$ compound, we also attribute the dielectric anomaly (the kink observed in the $\varepsilon_{\mathrm{r}}^{\prime}(T)$ curves) to the dynamical deformation and/or motion of the imidazolium cations. ${ }^{28}$ Nevertheless, as this cation is more strongly linked to the framework through two linear H-bonds from low to room temperature, as shown by single crystal X-ray diffraction, we suggest that the increase of $\varepsilon_{\mathrm{r}}^{\prime}$ is probably related to the sporadically broken up of the antiparallel arrangement of the electrical dipoles of the imidazolium cations. Such vibrational mode would be only active for $T>200 \mathrm{~K}$, as supported by the results of the Raman studies and also by the large thermal displacement found for the whole imidazolium cations at room temperature (see Fig. S1 of ESI†).

Also, we suggest that a second sharper dielectric anomaly is expected to appear around 435 $\mathrm{K}$, associated to the complete disorder of the imidazolium cation, even if experimental restrictions of our experimental equipment does not allow us to measure at higher temperatures.

Finally, for a more complete dielectric characterization of the low-temperature phase of $\left(\mathrm{C}_{3} \mathrm{~N}_{2} \mathrm{H}_{5}\right)\left[\mathrm{Mn}(\mathrm{HCOO})_{3}\right]$ and taking into account that it consists of an antiferroelectric crystal structure composed of two sublattices (with two crystallographically different imidazolium cations) spontaneously polarized in antiparallel directions, we have carried out polarizationversus electric field measurements trying to confirm its electrically ordered nature. As it can be seen in Fig. 5, a linear relationship between $P$ and $E$ is observed, indicating a paraelectric response, instead of the double hysteresis loop expected for an antiferroelectric material. To understand this result it is worth remembering that the polarity of this compound is attributable to the local dipole moment associated to the imidazolium cations. Therefore for this material to exhibit antiferroelectric behaviour, the polarization should be "switchable" and "reversible" in the presence of an external electrical field. The reversal of the macroscopic polarization requires substantial rearrangements of the imidazolium cations, as well as $\mathrm{H}$-bond breaking. Therefore, this process requires probably larger energy than provided by the here applied external field to occur. 


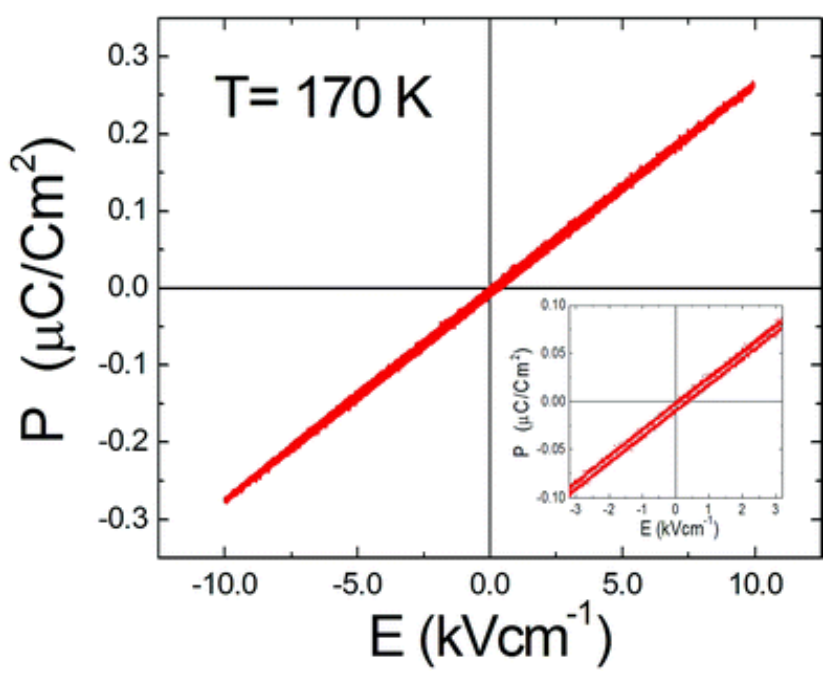

Fig. 5 Polarization $(P)$ versus electric field $(E)$ for the $\left(\mathrm{C}_{3} \mathrm{~N}_{2} \mathrm{H}_{5}\right)\left[\mathrm{Mn}(\mathrm{HCOO})_{3}\right]$ compound, measured at $50 \mathrm{~Hz}$ and 170 K. Inset: detail of the obtained curve in the low electric field region.

\section{Magnetic properties}

The main results of the magnetic studies carried out on this compound are shown in Fig. 6 and 7 (magnetic susceptibility data) and Fig. 8 (hysteresis loop). At low temperatures, the magnetization is seen to be both field and history dependent. For example, when measured under high magnetic fields ( $H \geq 1000$ Oe, Fig. 6), both the FC and $\mathrm{ZFC} \chi_{\mathrm{m}}(T)$ curves slightly diverge while showing a peak at $T \sim 9 \mathrm{~K}$, which reminds the typical signature for an antiferromagnetic transition at the Néel temperature.

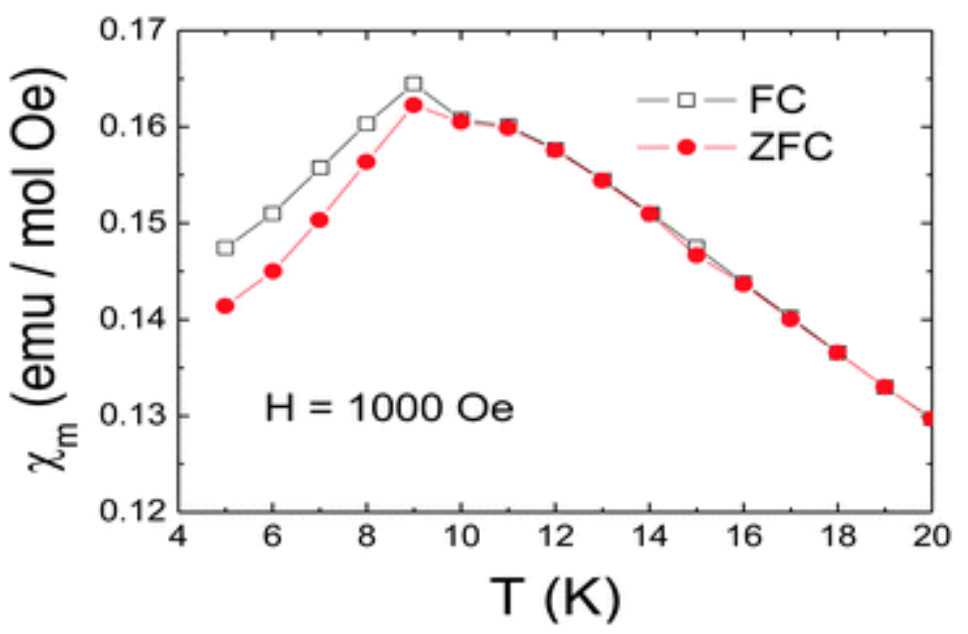

Fig. 6 ZFC-FC molar magnetic susceptibility of $\left(\mathrm{C}_{3} \mathrm{~N}_{2} \mathrm{H}_{5}\right)\left[\mathrm{Mn}(\mathrm{HCOO})_{3}\right]$ measured under a high applied magnetic field $H=1000 \mathrm{Oe}$. 


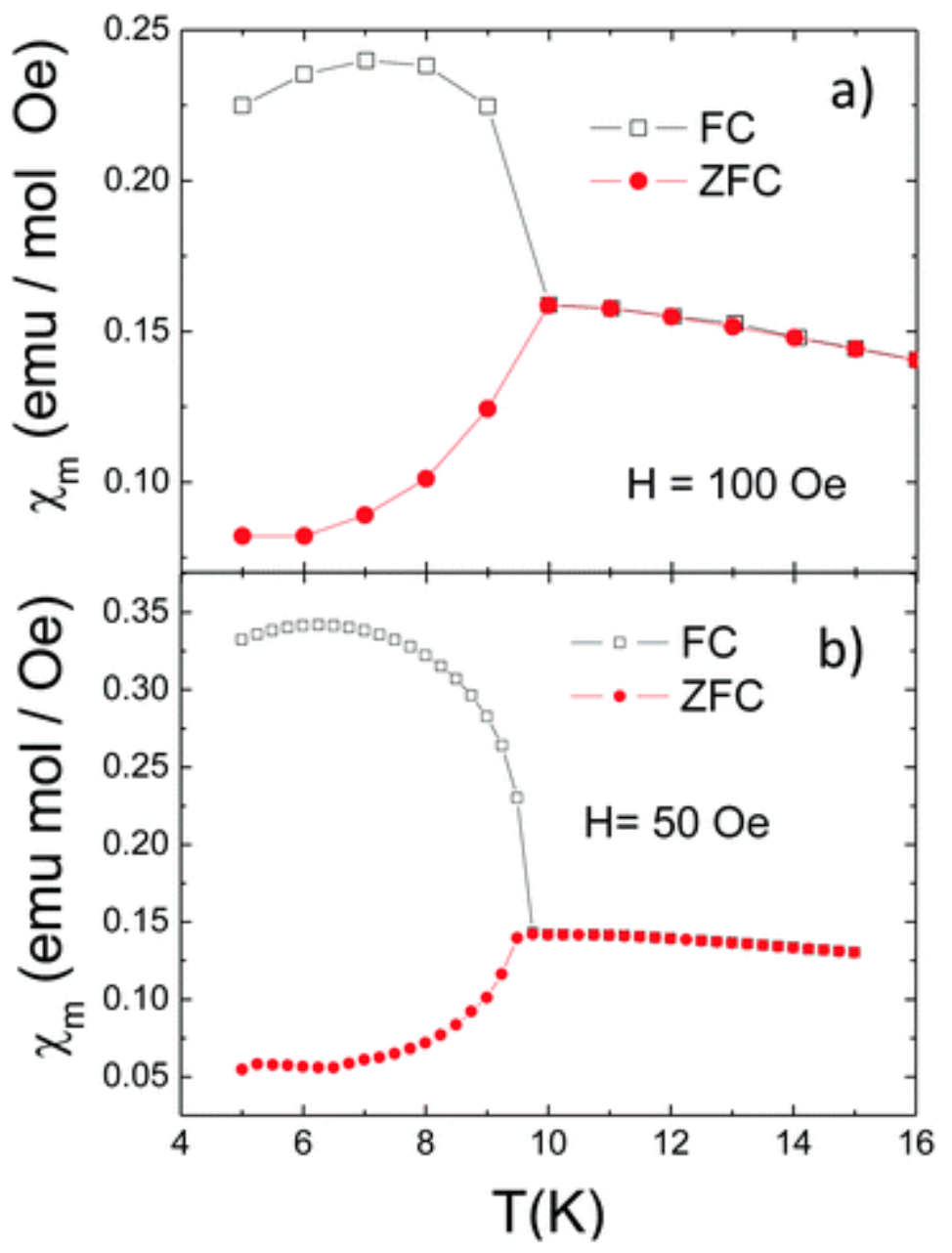

Fig. 7 ZFC-FC molar magnetic susceptibility of $\left(\mathrm{C}_{3} \mathrm{~N}_{2} \mathrm{H}_{5}\right)\left[\mathrm{Mn}(\mathrm{HCOO})_{3}\right]$ measured under low applied magnetic fields: (a) $H=100 \mathrm{Oe}$ and (b) $H=50$ Oe.

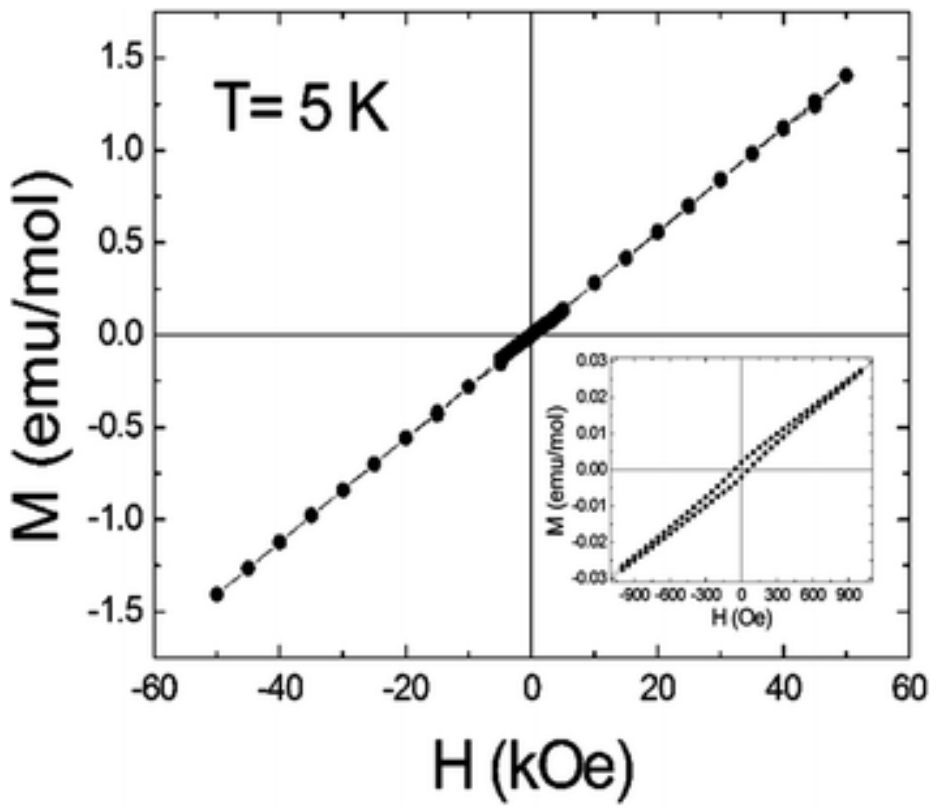

Fig. $8 \mathrm{ZFC}$ magnetization versus applied field measured at $T=5 \mathrm{~K}$ for the $\left(\mathrm{C}_{3} \mathrm{~N}_{2} \mathrm{H}_{5}\right)\left[\mathrm{Mn}(\mathrm{HCOO})_{3}\right]$ compound. Inset: detail of the obtained curve in the low magnetic field region.

Meanwhile, the ZFC and FC $\chi_{\mathrm{m}}(T)$ curves measured under low magnetic fields (50 Oe, 100 Oe) diverge below $T \sim 9 \mathrm{~K}$, and FC curve increase upon further cooling (see Fig. 7). 
The observed field dependence of the magnetization for this compound suggests the presence of weak ferromagnetism or canted antiferromagnetism, probably due to the presence of a non-centrosymmetric $\mathrm{HCOO}^{-}$ligand between two magnetic ions, which allows the occurrence of Dzyaloshinsky-Moriya (DM) interactions or antisymmetric exchanges, and thus spin canting. $^{2}$

Linear fitting of the $\chi_{\mathrm{m}}{ }^{-1} v$ s. $T$ data above $20 \mathrm{~K}$ affords very good agreement to a CurieWeiss behaviour (see Fig. S5 of ESI $\dagger$ ) from which a value of $C=4.59 \mathrm{~cm}^{3} \mathrm{Kmol}^{-1}$, a $\mu_{\text {eff }}=$ $5.54 \mu_{\mathrm{B}}$ and a $\theta=-14 \mathrm{~K}$ are calculated, where the symbol $C, \mu_{\mathrm{eff}}$ and $\theta$ have their usual meanings. The obtained values are very close to those expected for a $\mathrm{Mn}^{2+}$ cation with $S=5 / 2$ and $g=2.00\left(\mu_{\text {cal. }}=5.92 \mu_{\mathrm{B}}\right)$. Also, the observed negative Weiss constant corresponds to the antiferromagnetic exchange interactions between the nearest-neighbour metal ions as also found in other systems where the $\mathrm{Mn}^{2+}$-ions are connected through formate bridges in an anti-anti coordination mode. ${ }^{12 a}$

Furthermore, taking into account that, in this context, the three-dimensional nature of this compound could be simplified to a simple cubic cell, we have adjusted the obtained susceptibility data to the model of Rushbrooke and $\mathrm{Wood}^{29}$ for simple cubic Heisenberg antiferromagnetic systems:

$$
\chi=\frac{35}{12} \frac{N \mu_{\mathrm{B}}^{2}}{k} \frac{g^{2}}{T}\left(1+\sum_{n=1}^{6} C_{n} x^{n}\right)
$$

where $x=J / k T, C_{1}=35, C_{2}=221.67, C_{3}=608.22, C_{4}=26049.66, C_{5}=210986, C_{6}=8014$ 980 and the rest of the symbols $N, \mu_{\mathrm{B}}, k, g$ and $J$ have their usual meanings. As it can be seen in Fig. S6 of ESI, $\uparrow$ the fitting is very good and yields a $J$ value of $-0.206(3) \mathrm{cm}^{-1}$ and $g=2.036(4)$. These values are consistent with those reported for $\mathrm{Mn}^{2+}$ ions bridged by anti-anti $\mathrm{HCOO}^{-}$ligands. ${ }^{12 \mathrm{a}, 30}$

In Fig. 8 we represent the isothermal magnetization of this compound as a function of a magnetic field, $M(H)$, measured at $5 \mathrm{~K}$. For high magnetic fields, the $M(H)$ data display an almost linear behaviour. Nevertheless, in the inset of the Fig. 8, we can see that the compound shows a small hysteresis loop, with very low coercive field and remanent magnetization $\left(H_{\mathrm{c}} \approx 5\right.$ Oe and $M_{\mathrm{r}} \approx 2.510^{-3} \mathrm{emu} \mathrm{mol}^{-1}$ ).

Taking into account that for weak ferromagnetism arising from spin canting, the canting angle $\alpha$ is related to $M_{\mathrm{R}}$ and $M_{\mathrm{S}}$ through $\sin (\alpha)=M_{\mathrm{R}} / M_{\mathrm{S}}$, it can be estimated for this compound a 
canting angle of $0.097^{\circ}$. The obtained values are very close to those previously reported for related $[\mathrm{AmineH}]\left[\mathrm{Mn}\left(\mathrm{HCOO}_{3}\right)\right]$ compounds. ${ }^{12}$

Therefore this compound, that presents a 3D long range canted antiferromagnetic arrangement below $T_{\mathrm{N}}=9 \mathrm{~K}$ which coexist with the just described antiferroelectric order, is a new example of a perovskite-based hybrid inorganic-organic with multiferroic properties.

\section{Conclusions}

In this work we deepen in the structure and properties of the $\left(\mathrm{C}_{3} \mathrm{~N}_{2} \mathrm{H}_{5}\right)\left[\mathrm{Mn}(\mathrm{HCOO})_{3}\right]$ compound, a Mn-formate framework that, despite the big size of the five-membered ring imidazolium guest located inside its cavities, displays a perovskite-like structure with $4^{12} \cdot 6^{3}$ topology. In particular, we focus in its low-temperature phase, that shows a monoclinic structure, and we study its dielectric and magnetic properties.

In this low-temperature phase (S.G. $P 2_{1} / n$ ) the imidazolium cations, that sit oblique within the cavities of the framework structure, are cooperatively ordered inside the pseudo-cubo octahedral cavities and the $\mathrm{Mn}^{2+}$ cations show out-of-center octahedral distortions. Nevertheless in the resulting centrosymmetric crystal structure, the associated electric dipole moments present an antiparallel arrangement and cancel each other so that this compound with an antiferroelectric crystal structure displays a zero net polarization.

In addition, comparison of the structural, Raman and dielectric data corresponding to this low-temperature phase and obtained at different temperatures, reveals the presence of a certain degree of dynamical motion of the entire imidazolium cations for temperatures above $\sim 220 \mathrm{~K}$.

This seems to be the origin of the anomaly already detected in the $\varepsilon_{\mathrm{r}}(T)$ measurements at $T \approx$ $220 \mathrm{~K}$, as above that temperature thermal energy seems to be able to sporadically break the antiferroelectric ordering associated to the imidazolium cations, resulting in a linear increase of its dielectric constant with temperature.

In addition, this compound shows canted antiferromagnetism below $T_{\mathrm{N}}=9 \mathrm{~K}$ with a slightly non-collinear arrangement of the magnetic moments which yields to weak ferromagnetism.

Therefore, $\left(\mathrm{C}_{3} \mathrm{~N}_{2} \mathrm{H}_{5}\right)\left[\mathrm{Mn}(\mathrm{HCOO})_{3}\right]$ is an example of a new multiferroic material that exhibits coexistence of magnetic and electric ordering.

\section{Acknowledgements}

The authors are grateful for financial support from Ministerio de Economía y Competitividad MINECO (Spain) under project FEDER MAT2010-21342-C02-01 and from Xunta de Galicia 
under project PGIDIT10PXB103272PR. B.P.-D. also wants to thank MICINN for a FPI fellowship.

\section{Notes and references}

1. A. K. Cheetham and C. N. R. Rao, Science, 2007, 318, 58-59

2. Z. Wang, K. Hu, S. Gao and H. Kobayashi, Adv. Mater., 2010, 22, 1526-1533

3. P. Jain, N. S. Dalal, B. H. Toby, H. W. Kroto and A. K. Cheetham, J. Am. Chem. Soc., 2008, 130, 10450-10451

4. P. Jain, V. Ramachandran, R. J. Clark, H. D. Zhou, B. H. Toby, N. S. Dalal, H. W. Kroto and A. K. Cheetham, J. Am. Chem. Soc., 2009, 131, 13625-13627

5. G.-C. Xu, W. Zhang, X.-M. Ma, Y.-H. Chen, L. Zhang, H.-L. Cai, Z.-M. Wang, R.-G. Xiong and S. Gao, J. Am. Chem. Soc., 2011, 133, 14948-14951

6. (a) Z. Wang, B. Zhang, K. Inoue, H. Fujiwara, T. Otsuka, H. Kobayashi and M. Kurmoo, Inorg. Chem., 2007, 46, 437-445 ; (b) G.-C. Xu, X.-M. Ma, L. Zhang, Z.-M. Wang and S. Gao, J. Am. Chem. Soc., 2010, 132, 9588-9590 .

7. Z. Wang, B. Zhang, T. Otsuka, K. Inoue, H. Kobayashi and M. Kurmoo, Dalton Trans., 2004, 2209-2216.

8. K.-L. Hu, M. Kurmoo, Z. Wang and S. Gao, Chem.-Eur. J., 2009, 15, 12050-12064 .

9. Z. Wang, X. Zhang, S. R. Batten, M. Kurmoo and S. Gao, Inorg. Chem., 2007, 46, 84398441 .

10. M. Sánchez-Andújar, S. Presedo, S. Yáñez-Vilar, S. Castro-García, J. Shamir and M. A. Señarís-Rodríguez, Inorg. Chem., 2010, 49, 1510-1516 .

11. B. Pato-Doldán, M. Sánchez-Andújar, L. C. Gómez-Aguirre, S. Yáñez-Vilar, J. LópezBeceiro, C. Gracia-Fernández, A. A. Haghighirad, F. Ritter, S. Castro-García and M. A. Señarís-Rodríguez, Phys. Chem. Chem. Phys., 2012, 14, 8498-8501

12. (a) X.-Y. Wang, L. Gan, S.-W. Zhang and S. Gao, Inorg. Chem., 2004, 43, 4615-4625 ; (b) N. H. M. Hcoo, M. Ni, P. J. Baker, T. Lancaster, I. Franke, W. Hayes, S. J. Blundell, F. L. Pratt, P. Jain, Z. Wang and M. Kurmoo, Phys. Rev. B: Condens. Matter Mater. Phys., 2010, 82, 12407-12411. 
13. B. Zhou, Y. Imai, A. Kobayashi, Z.-M. Wang and H. Kobayashi, Angew. Chem., Int. Ed., 2011, 50, 11441-11445.

14. A. Stroppa, P. Jain, P. Barone, M. Marsman, J. M. Perez-Mato, A. K. Cheetham, H. W. Kroto and S. Picozzi, Angew. Chem., Int. Ed., 2011, 50, 5847-5850 .

15. B.-Q. Wang, H.-B. Yan, Z.-Q. Huang and Z. Zhang, Acta Crystallogr., Sect. C: Cryst. Struct. Commun., 2013, 69, 616-619 .

16. G. M. Sheldrick, SHELXS-97: Program for Crystal Structure Resolution, University of Göttingen, Göttingen, Germany, 1997 .

17. G. M. Sheldrick, SHELXL-97: Program for Crystal Structure Analysis, University of Göttingen, Göttingen, Germany, 1997 .

18. A. C. Larson and R. B. von Dreele General, Structure Analysis System (GSAS), Los Alamos National Laboratories, Report LAUR, 1990, pp. 86-748 .

19. J. R. Macdonald, LEVM version 8.0 Comlex Nonlinear Squares Fitting Program, 2003.

20. G. A. Bain and J. F. Berry, J. Chem. Educ., 2008, 85, 532-536 .

21. P. S. Halasyamani, Chem. Mater., 2004, 16, 3586-3592 .

22. A. Piecha, R. Jakubas, G. Bator and J. Baran, Vib. Spectrosc., 2009, 51, 226-237.

23. A. Piecha, R. Jakubas, A. Pietraszko, J. Baran, W. Medycki and D. Kruk, J. Solid State Chem., 2009, 182, 2949-2960 .

24. A. Piecha, R. Jakubas, A. Pietraszko and J. Baran, J. Mol. Struct., 2007, 844-845, $132-$ 139 .

25. W. Zhang, Y. Cai, R.-G. Xiong, H. Yoshikawa and K. Awaga, Angew. Chem., Int. Ed., 2010, 49, 6608-6610 .

26. D. C. Sinclair, T. B. Adams, F. D. Morrison and A. R. West, Appl. Phys. Lett., 2002, 80, 2153-2155.

27. (a) J. F. Scott, J. Phys.: Condens. Matter, 2008, 20, 21001 ; (b) A. Loidl, S. Krohns, J. Hemberger and P. Lunkenheimer, J. Phys.: Condens. Matter, 2008,20, 19100 .

28. We have to note that the $\left[\mathrm{Mn}(\mathrm{HCOO})_{3}\right]^{-}$framework would also contribute to the dielectric response, even if its contribution would be much smaller than that of the imidazolium cations that display a large electric dipole moment. 
29. G. S. Rushbrook and P. J. Wood, Mol. Phys., 1958, 1, 257-283 .

30. M. Viertelhaus, H. Henke, C. E. Anson and A. K. Powell, Eur. J. Inorg. Chem., 2003, 2283-2289.

\section{Footnotes}

$\uparrow$ Electronic supplementary information (ESI) available: Structural details; results of Le Bail refinement of powder X-ray diffraction; DSC curve; fits of the $\chi \mathrm{m}(\mathrm{T})$ data; observed frequencies in $\mathrm{cm}^{-1}$ of the Raman spectra at $100 \mathrm{~K}$ and suggested assignments. The cif files with the structural information obtained by single crystal X-ray diffraction. CCDC 959632 and 959633. For ESI and crystallographic data in CIF or other electronic format see DOI:10.1039/c3ra43165g

\$ (CCDC 959633): Monoclinic, $\mathrm{a}=12.1962(3) \AA, \mathrm{b}=12.3675(3) \AA, \mathrm{c}=12.8155(3) \AA, \alpha=$ $90.00^{\circ}, \beta=91.4280(10)^{\circ}, \gamma=90.00^{\circ}, \mathrm{V}=1932.44(8) \AA 3, \mathrm{~T}=100(2) \mathrm{K}$, space group $\mathrm{P} 21 / \mathrm{n}, \mathrm{Z}=$ 8, 20812 reflections measured, 4863 independent reflections $($ Rint $=0.0344)$. The final R1 values were $0.0341(\mathrm{I}>2 \sigma(\mathrm{I}))$. The final $w R(F 2)$ values were $0.0873(\mathrm{I}>2 \sigma(\mathrm{I}))$. The final $\mathrm{R} 1$ values were 0.0450 (all data). The final $w R(F 2)$ values were 0.0936 (all data). The goodness of fit on F2was 1.081 .

$\S($ CCDC 959632): Monoclinic, $\mathrm{a}=12.3034(4) \AA ̊ ., \mathrm{b}=12.4408(4) \AA \mathrm{A}, \mathrm{c}=12.8156(4) \AA, \alpha=$ $90.00^{\circ}, \beta=91.190(2)^{\circ}, \gamma=90.00^{\circ}, \mathrm{V}=1961.19(11) \AA 33, \mathrm{~T}=296(2) \mathrm{K}$, space group $\mathrm{P} 21 / \mathrm{n}, \mathrm{Z}=$ 8, 20976 reflections measured, 4899 independent reflections (Rint $=0.0376)$. The final R1 values were $0.0381(\mathrm{I}>2 \sigma(\mathrm{I}))$. The final $w R(F 2)$ values were $0.1042(\mathrm{I}>2 \sigma(\mathrm{I}))$. The final $\mathrm{R} 1$ values were 0.0668 (all data). The final $w \mathrm{R}(\mathrm{F} 2)$ values were 0.1251 (all data). The goodness of fit on F2was 0.905 . 